\title{
Prognostic indicators of Disseminated Intravascular Coagulation in pregnancy
}

\author{
Dr.NehaGupta, Dr.Sudeep Nagori, Dr.Jitendra Kumar, Dr.Gayatri Nagori
}

\begin{abstract}
Objective: The aim of this work is to evaluate the underlying causes, its complications, and effect of various DIC parameters on maternal outcomes.

Method: This is prospective cum retrospective study.It included all Patients who developed DIC in pregnancy and postpartum in period of 2 years.

Statistical analysis: Mean and percentage were used for presentation of the data.

Result: The mean age of DIC patient is 27.35 3.11 SD and mean of gestational age is $34 \pm 3.9$ week. This study show maximum no. of patients with PPH developed DIC. This may be the cause or consequences of DIC. Then next important cause is Preeclampsia, Septicemia, Acute fatty liver and IUD. DIC itself is a complication of various obstetrics condition but it also lead to various complications such as bleeding, renal dysfunction, liver dysfunction, respiratory dysfunction, shock, thromboembolic phenomenon and death. The diagnosis of DIC relies on clinical manifestations and laboratory tests. The diagnosis of DIC cannot made on a single laboratory value, but rather the constellation of laboratory markers and a consistent history of an illness known to cause DIC. FDP and D-Dimer are sensitive marker for diagnosis of DIC. From the present study it is concluded that DIC has high maternal (16\%) mortality rate.

Conclusion: Early diagnosis with prompt treatment, including a quick decision for surgical intervention, and eradication of predisposing conditions would minimize maternal morbidity and mortality. The treatment is urgent.
\end{abstract}

Keywords:Disseminated intravascular coagulation, Fibrin degredation product

\section{Introduction}

The dangers surrounding obstetrical disseminated intravascular coagulation were recognized and described as early as 1901 in the obstetrical literature, when Joseph De Lee first reported a fatal case of hemorrhagic diathesis with placental abruption, hypothesizing, "Is there such a disease as acquired hemophilia?... I believe there is such an affection as a temporary hemophilia, but the demonstration of the same, I admit, presents no little difficulty." ${ }^{[1]}$ As obstetrical hemorrhage is still the leading cause of maternal death. As activation of the coagulation pathway is a physiological response to injury, the development of disseminated intravascular coagulation (DIC) is a warning signal to the clinician that the primary pathological disease state is decompensating. In a study of discharge coding data from the United States Nationwide In patient Sample (NIS), the prevalence of DIC related to pregnancy increased by 35.5 percent between 1998 and 2009, from 9.2 to 12.5 per 10,000 delivery hospitalizations, and DIC accounted for approximately one-quarter of maternal deaths during that period. ${ }^{[2]}$ Peripartum hemorrhage is a common cause of DIC in pregnant women. It is estimated to account for 1 to 5 percent of all cases of DIC in high-resource countries, and an even higher proportion of DIC cases in pregnancy, DIC can occur in several settings, which include emergencies such as placental abruption and amniotic fluid embolism as well as complications such as pre-eclampsia. ${ }^{[3,4]}$ The prognosis for disseminated intravascular coagulation varies according to each individual patient. It depends on the cause of disseminated intravascular coagulaion. Routinely performed tests for DIC such as platelet count, Prothrombin time, INR, APTT and FDP/D- Dimer. There is no single test that would diagnose DIC. Therapy of DIC aims at treating the primary cause. Early diagnosis with prompt treatment, including a quick decision for surgical intervention, and eradication of predisposing conditions would minimize maternal morbidity and mortality. There is no doubt that the major determinant of survival is our ability to identify the underlying trigger and manage it successfully. The objectives of this study were to determine the antecedent factors, morbidity, and mortality associated with DIC and the effect of various parameters on the outcome of DIC in pregnant patients and its complication in a tertiary obstetrical hospital population over the two years period.

\section{Aims And Objectives}

- To evaluate various clinical and laboratory indicators in term of morbidity and mortality in maternal disseminated intravascular coagulation.

- To assess the various causes of disseminated intravascular coagulation in pregnancy.

- To predict the various complication of disseminated intravascular coagulation in pregnancy. 


\section{Materials And Methods}

This study included all Patients who developed DIC in pregnancy and postpartum period and admitted to the Choithram Hospital and Research in period of 2 years .A retrospective cum prospective study was conducted in Department of Obstetrics and Gynaecology . For the retrospective part of the study, the medical records of all patients with DIC in pregnancy was reviewed. Data was collected and recorded in a predesigned case proforma and analyzed. For the prospective part of the study, all the prospective subjects were identified with diagnosis of DIC in pregnancy and the informed consent was taken. Detailed history, thorough examination, investigation, and management was done and maternal and fetal outcome was evaluated. Data was collected and recorded in a predesigned case proforma and analyzed.

Inclusion Criteria- The population was comprise of all women in their pregnancy and postpartum period uptill 42 days period who had presented with DIC either clinically or were having impaired DIC parameters. The affected person was often acutely ill and shocked with widespread hemorrhage (common bleeding sites are mouth, nose, vaginal and venipuncture sites), extensive bruising, and one or more organ failure, and had any one or more impaired DIC parameters [platelet $<1.5 \mathrm{lac}$, PTINR $>1.5$, FDP $>10 \mathrm{mcg} / \mathrm{dl}$, DDimer $>0.5 \mathrm{mcg} / \mathrm{ml}$, fibrinogen level $<100 \mathrm{mg} / \mathrm{dl}]$ in pregnancy and postpartum period.

Exclusion Criteria- All other obstetric patients antenatal postnatal till 42 days of delivery admitted to the obstetrics department with other diagnosis and didn't have clinical and laboratory pictures of DIC. Patients were also excluded who came with diagnosis of other coagulopathies (hereditary coagulation disorder), and gestational thrombocytopenia with no other coagulation abnormalities.Patients those were not willing to provide voluntary written informed consent for participation in the study.

\section{Statistical Analysis Used}

Mean and percentage were used for presentation of the data.

\section{Observations And Results}

Out of 31 cases, 28 (84\%) patients were between age group 21-30 years, 4 (13\%) patients were between $31-40$,and only $1(3.2 \%)$ patient were $<20$ years. The mean age of patient is $27.35 \pm 3.11$ SD.All the cases were unbooked and referred from other hospitals. Equal no. of patients were found between 30-36 weeks of pregnancy and $>36$ week of pregnancy i.e. 13 (42\%) patients. And only 4 (16.1\%) patients were $<30$ week of pregnancy developed DIC in pregnancy. The mean of gestational age is $34 \pm 3.9$ weeks of pregnancy. The common causes of DIC were shown in Table 1.Post partum Hemorrhage in $12(38.7 \%)$ DIC patients, it might be the cause or consequences of DIC. Next common causes were abruptio in $9(29 \%)$ patients, eclampsia and HELLP in $7(22 \%)$ patients. Severe Preeclampsia in $3(9.7 \%)$ patients. IUD also leads to DIC in 4 (12.9\%) patients. And $3(9.7 \%)$ patients developed septicemia and $3(9.7 \%)$ patients developed acute fatty liver in pregnancy which also causes DIC.

Table No. 1: Distribution of patients according to risk factors

\begin{tabular}{|l|l|l|}
\hline Risk factors & Number & Percentage \\
\hline Abruptio & 9 & 29.00 \\
\hline Eclampsia + HELLP & 7 & 22.60 \\
\hline Preeclampsia & 3 & 9.70 \\
\hline PPH & 12 & 38.70 \\
\hline Septicemia & 3 & 9.70 \\
\hline Acute fatty liver & 3 & 9.70 \\
\hline IUD & 4 & 12.90 \\
\hline
\end{tabular}

Table No 2 showing the distribution of DIC patients according to PT INR, there were 15 (48.4\%) patients were having PT INR in range of 1.5-3 and all were recovered, showed good maternal outcome. There were $14(45.2 \%)$ patients having PT INR in range of 3-6,out of which 3 were died, showing $21 \%$ mortality rate. 2 patients were showing INR $>6$, and both died due to DIC and its complications, showing $100 \%$ mortality rate. Table No 3 showing 12 (38.71\%) patients were having platelet count <50,000/cumm out of which $4(25 \%)$ were died. Maximum DIC patients (18 cases) were having platelet count in range of 50,000-1,00,000/cumm of which only $1(5.5 \%)$ were died. This shows that those having low platelet count were having poor maternal outcome and high DIC scoring. All the patients were having FDP $>40$ (normal value $<10$ ) and D-DIMER $>4$ (normal value $<0.5$ ) which were sensitive marker for diagnosis of DIC.

Table No. 2: Distribution of patients according to PT INR and Its Maternal Outcome

\begin{tabular}{|l|l|l|l|l|}
\hline PT INR & Number & Percentage & Maternal Outcome & \\
\cline { 4 - 5 } & & & Died & Recovered \\
\hline$<3.0$ & 15 & 48.40 & 0 & 15 \\
\hline $3.0-6.0$ & 14 & 45.20 & 3 & 11 \\
\hline$>6.0$ & 2 & 6.50 & 2 & 0 \\
\hline Total & 31 & 100.00 & 5 & 26 \\
\hline
\end{tabular}


Table No. 3: Distribution of patients according to Platelet Count and Its Maternal Outcome

\begin{tabular}{|l|l|l|l|l|}
\hline Platelet Counts & Number & Percentage & Maternal Outcome \\
\cline { 4 - 5 } (/cumm) & & & Died & Recovered \\
\hline$<50000$ & 12 & 38.71 & 4 & 8 \\
\hline $50000-100000$ & 18 & 58.07 & 1 & 17 \\
\hline$>100000$ & 1 & 3.22 & 0 & 1 \\
\hline Total & 31 & 100.00 & 5 & 26 \\
\hline
\end{tabular}

In the present study the complications developed in DIC patients were Post partum hemorrhage in 10 $(32.33 \%)$ patients, of which 2 were died, 8 (25.8\%) patients developed Acute Renal Failure, of which 3 were died, showing poor maternal outcome $.3(9.7 \%)$ patients developed Pleural effusion, of which 2 were died due to ARDS..5 (16.1\%) patients developed Liver dysfunction and all were revived. 4 (13\%) patient came with Shock, out of which $50 \%$ patients died. All DIC patients were managed in ICU initially. The maternal mortality rate in DIC patients in present study is $16.1 \%$ (5/31), showing DIC has high mortality rate in obstetrics patients. By aggressive management, we were able to save 26 patients.

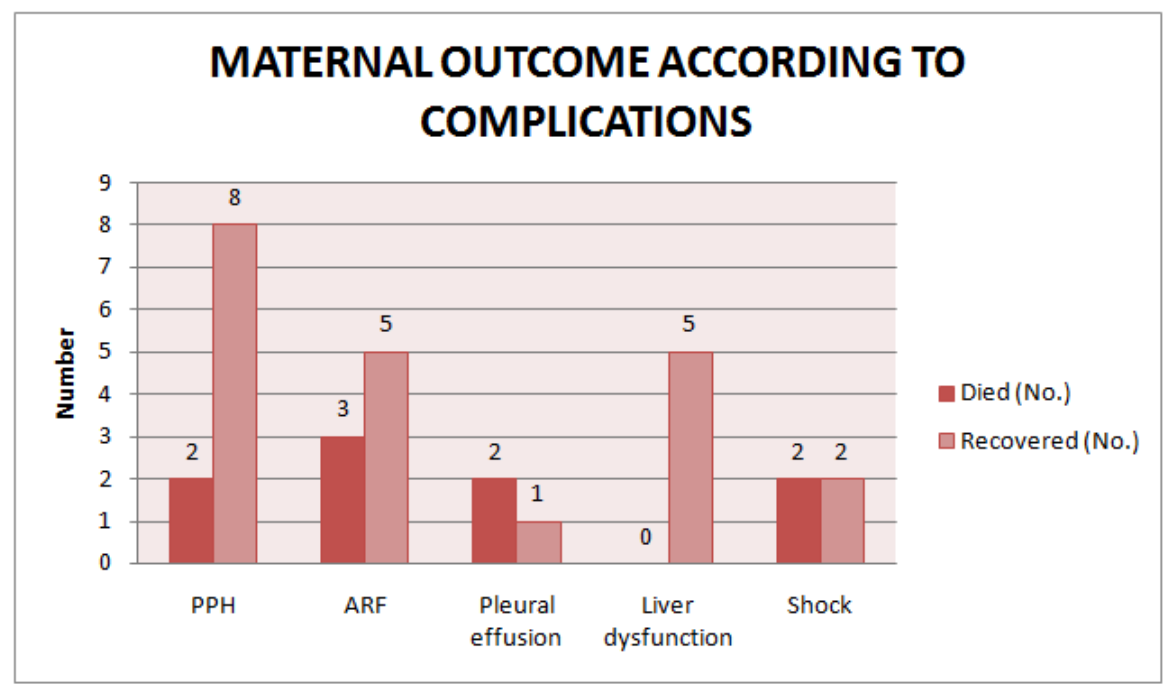

\section{Discussion}

Disseminated intravascular coagulation (DIC) is a challenge to obstetricians. It is well described that DIC in pregnancy results in very high maternal morbidity and mortality. In the present study, the mean age of DIC patients in is $27.35 \pm 3.11$ years and mean of mean gestational age is $34 \pm 3.9$ weeks of pregnancy. which is almost similar to study of Darrien D.et al (2012), ${ }^{[5]}$ in which the average age of DIC patient in pregnancy were 28.1 years and the average gestational age was found 35.4 weeks of gestation. FDP and D-Dimer are sensitive marker for diagnosis of DIC. Erez O et al. ( 2014), ${ }^{[6]}$ found that a PT difference $\geq 1.55$ had an $87 \%$ sensitivity and $90 \%$ specificity for the diagnosis of DIC, the platelet count had an $86 \%$ sensitivity and $71 \%$ specificity for the diagnosis of DIC, fibrinogen concentrations had a cut-off point $\leq 3.9 \mathrm{~g} / \mathrm{L}$ had a sensitivity of $87 \%$ and a specificity of $92 \%$ for the development of DIC In the present study, maternal mortality rate is $16.1 \%(5 / 31)$, indicating DIC is associated with high mortality rate in obstetrics patients. However Darrien D. et al (2012) . ${ }^{[5]}$ reported three maternal deaths, a case fatality rate of 1 in 16 and mortality rate of $6.25 \%$. On contrary, Koranantakul ${ }^{1}$ et al (2007), ${ }^{[7]}$ reported that 6 out of 25 patients were died, giving a case mortality rate of $24 \%$. AYang M et al (1999) ${ }^{[8]}$ reported the mortality rate of $26.9 \%$. A study conducted by Zhenjiang, et al (2002). ${ }^{[9]}$ found the similar maternal mortality rate $21.05 \%$.

\section{Conclusion}

DIC is an acquired syndrome characterized by the intravascular activation of coagulation with loss of localization arising from different causes. At present the diagnosis of DIC relies on clinical manifestations and laboratory tests. The diagnosis of DIC cannot made on a single laboratory value, but rather the constellation of laboratory markers and a consistent history of an illness known to cause DIC. Laboratory markers of DIC such as PT INR, Platelet count, FDP and D-dimer which are useful to predict the maternal morbidity and mortality. Those who have extremely deranged DIC parameters have poor maternal outcome. All these indicators are used for DIC scoring, those who have high DIC scoring have poor maternal outcome. The present study suggested that a combination of the various laboratory indicators, predicts mortality in obstetrics DIC patients. 
DIC itself is a complication of various obstetrics condition but it also lead to various complications such as bleeding, renal dysfunction, liver dysfunction, respiratory dysfunction, shock, thromboembolic phenomenon and death. From the present study it is concluded that DIC has high maternal (16\%) mortality rate. Early diagnosis with prompt treatment, including a quick decision for surgical intervention, and eradication of predisposing conditions would minimize maternal morbidity and mortality. The treatment is urgent.

Acknowledgement We thank the medical director of Choithram Hospital, HOD and all consultants of obstetrics and gynaecology department for their cooperation.

Conflictofinterest The authors declare they have no conflict of interest to disclose Contribution to authorship Authors make substantial contributions to acquisition of data and analysis and interpretation of data and give final approval of version to be submitted

\section{References}

[1]. DeLee JB. A case of fatal hemorrhagic diathesis, with premature detachment of the placenta. Am J Obstet Dis Women Child 1901;44:785-92.

[2]. Callaghan WM, Creanga AA, Kuklina EV. Severe maternal morbidity among delivery and postpartum hospitalizations in the United States. ObstetGynecol 2012;120:1029.7.

[3]. StirlingY,Woolf L, North WR. Haemostasis in normal pregnancy. Thromb Haemost 1984;52:176 \pm 182

[4]. Gilabert J, Aznar J, Parilla J. Alteration in the coagulation and fibrinolysis system in pregnancy, labour and puerperium, with special reference to a possible transitory state of intravascular coagulationduringlabour. Thromb Haemost 1978;40:387-396.

[5]. Darrien D. Rattray, MD, Colleen M. O’Connell, PhD, Thomas F. Baskett, MB J Obstet Gynaecol Can 2012;34(4):341-347.

[6]. Erez,Novack L,Beer-Weisel R,Dukler D. DIC Score in Pregnant Women - A Population Based Modification of the International Society on Thrombosis and Hemostasis Score. Published: April 11, 2014.DOI: 10.1371/journal.pone.0093240

[7]. Kor-anantakul O, Lekhakula A. Overt disseminatedcoagulation in obstetric patients. J Med Assoc Thai 2007;90:857-64.

[8]. AYang M, Shen W, Chen L. Zhonghua Fu Chan Ke Za Zhi. 1999 Oct;34(10):600-2.;

[9]. Zhenjiang, Suzhou University, and Jiangsu People's Hospital, pregnancy induced hypertension and the relevance of DIC. Am J Obstet Gynecol 2002, 186:1105-1106. 